\title{
Crystal structure of $(1 \mathrm{a} \alpha, 2 \alpha, 5 \beta, 8 \alpha, 8 \mathrm{a} \alpha)$-2,3,8-trichloro-1a,2,5,6,8,8a- hexahydro-5-methoxy-9-oxo-2,8-methano-1H-cyclopropa[4,5]cyclohepta- $[1,2-b]$ pyran, $\left(\mathrm{C}_{9} \mathrm{H}_{4} \mathrm{OCl}_{3}\right)\left[\mathrm{C}_{3} \mathrm{H}_{4} \mathrm{O}\left(\mathrm{OCH}_{3}\right)\right]$
}

\author{
K. Peters ${ }^{*, I}$, E.-M. Peters ${ }^{1}$, M. Braun ${ }^{\text {II }}$ and M. Christ ${ }^{\text {II }}$ \\ I Max-Planck-Institut für Festkörperforschung, Heisenbergstraße 1, D-70506 Stuttgart, Germany \\ II Universität Würzburg, Institut für Organische Chemie, Am Hubland, D-97074 Würzburg, Germany
}

Received July 30, 1999, CCDC-No. 1267/247

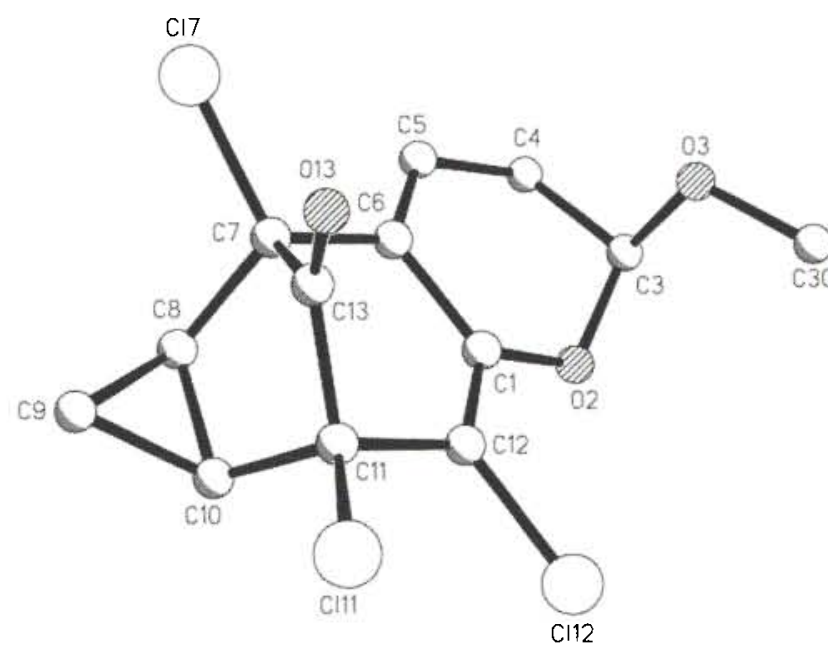

\begin{abstract}
$\mathrm{C}_{13} \mathrm{H}_{1 \mathrm{I}} \mathrm{Cl}_{3} \mathrm{O}_{3}$, monoclinic, $P 12_{1} / n 1$ (No. 14), $a=11.972(8) \AA$, $b=12.475(8) \AA, c=9.442(6) \AA, \beta=100.86(5)^{\circ}, V=1384.9 \AA^{3}$, $Z=4, R_{\mathrm{g}}(F)=0.049, w R(F)=0.045, T=293 \mathrm{~K}$.
\end{abstract}

\section{Source of materiai}

The title compound was prepared by irradiation of a solution of chloranil and cyclopropene in chlorobenzene, kept at $243 \mathrm{~K}$, with a Hanovia mercury lamp (medium pressure, $450 \mathrm{~W}$ ), surrounded by a glass filter to prevent passage of light of $\lambda<400 \mathrm{~nm}$, and subsequent treatment of the product with methanol at $298 \mathrm{~K}$ [1].

Table 1. Data collection and handling.

Crystal:

Wavelength:

$\mu$ :

Diffractometer, scan mode:

$2 \theta_{\max }$ :

$N(h k l)_{\text {measured, }} N(h k l)_{\text {unique: }}$

Criterion for $F_{\mathrm{obs}}, N(h k l)_{\mathrm{g}}$

$N(\text { param })_{\text {refined: }}$

Program: colourless prism, size $0.8 \times 0.8 \times 0.5 \mathrm{~mm}$ Mo $K_{\alpha}$ radiation $(0.71073 \AA)$

$6.60 \mathrm{~cm}^{-1}$

Nicolet R3m/V, Wyckoff

$55^{\circ}$

3507,3210

$F_{\text {obs }}>3 \sigma\left(F_{\text {obs }}\right), 2861$

172

SHELXTL-plus [2]

Table 2. Atomic coordinates and displacement parameters (in $\ddot{A}^{L}$ ).

\begin{tabular}{llllll}
\hline Atom & Site & $x$ & $y$ & $z$ & $U_{\text {iso }}$ \\
\hline $\mathrm{H}(3 \mathrm{~A})$ & $4 e$ & $0.4126(3)$ & $0.6857(3)$ & $-0.0314(4)$ & 0.08 \\
$\mathrm{H}(4 \mathrm{~A})$ & $4 e$ & $0.3564(3)$ & $0.7391(3)$ & $-0.2622(4)$ & 0.08 \\
$\mathrm{H}(4 \mathrm{~B})$ & $4 e$ & $0.4283(3)$ & $0.6361(3)$ & $-0.2765(4)$ & 0.08 \\
$\mathrm{H}(5 \mathrm{~A})$ & $4 e$ & $0.5048(3)$ & $0.7605(3)$ & $-0.4183(3)$ & 0.08 \\
$\mathrm{H}(8 \mathrm{~A})$ & $4 e$ & $0.5442(3)$ & $1.0192(3)$ & $-0.3889(3)$ & 0.08 \\
$\mathrm{H}(9 \mathrm{~A})$ & $4 e$ & $0.7775(3)$ & $1.0995(3)$ & $-0.3357(3)$ & 0.08 \\
$\mathrm{H}(9 \mathrm{~B})$ & $4 e$ & $0.6802(3)$ & $1.1852(3)$ & $-0.3663(3)$ & 0.08 \\
$\mathrm{H}(10 \mathrm{~A})$ & $4 e$ & $0.6396(3)$ & $1.1273(3)$ & $-0.1129(3)$ & 0.08 \\
$\mathrm{H}(30 \mathrm{~A})$ & $4 e$ & $0.6601(3)$ & $0.5645(3)$ & $0.1148(4)$ & 0.08 \\
$\mathrm{H}(30 \mathrm{~B})$ & $4 e$ & $0.5362(3)$ & $0.5865(3)$ & $0.1393(4)$ & 0.08 \\
$\mathrm{H}(30 \mathrm{C})$ & $4 e$ & $0.6237(3)$ & $0.6809(3)$ & $0.1488(4)$ & 0.08 \\
& & & & & \\
\hline
\end{tabular}

Table 3. Atomic coordinates and displacement parameters (in $\AA^{2}$ ).

\begin{tabular}{|c|c|c|c|c|c|c|c|c|c|c|}
\hline Atom & Site & $x$ & $y$ & $z$ & $U_{11}$ & $U_{22}$ & $U_{33}$ & $U_{12}$ & $U_{13}$ & $U_{23}$ \\
\hline $\mathrm{Cl}(7)$ & $4 e$ & $0.68276(\overline{8})$ & $0.86334(8)$ & $-0.48375(9)$ & $0.0756(6)$ & $0.0824(7)$ & $0.0522(5)$ & $0.0095(5)$ & $0.0248(4)$ & $-0.0081(5)$ \\
\hline $\mathrm{Cl}(11)$ & $4 e$ & $0.85805(7)$ & $1.05139(8)$ & $0.02375(9)$ & $0.0587(5)$ & $0.0789(6)$ & $0.0649(6)$ & $-0.0193(5)$ & $-0.0038(4)$ & $0.0050(5)$ \\
\hline $\mathrm{Cl}(12)$ & $4 e$ & $0.65636(7)$ & $0.95688(8)$ & $0.16219(8)$ & $0.0669(5)$ & $0.0802(6)$ & $0.0443(4)$ & $0.0004(5)$ & $0.0141(4)$ & $-0.0028(4)$ \\
\hline $\mathrm{C}(1)$ & $4 \dot{e}$ & $0.5861(2)$ & $0.8629(2)$ & $-0.0924(3)$ & $0.041(2)$ & $0.050(2)$ & $0.051(2)$ & $-0.000(1)$ & $0.016(1)$ & $0.002(1)$ \\
\hline$O(2)$ & $4 e$ & $0.5080(2)$ & $0.8128(2)$ & $-0.0268(2)$ & $0.052(1)$ & $0.058(1)$ & $0.066(1)$ & $-0.006(1)$ & $0.027(1)$ & $-0.004(1)$ \\
\hline$C(3)$ & $4 e$ & $0.4729(3)$ & $0.7068(3)$ & $-0.0795(4)$ & $0.049(2)$ & $0.060(2)$ & $0.081(3)$ & $-0.008(2)$ & $0.025(2)$ & $0.002(2)$ \\
\hline$O(3)$ & $4 \dot{e}$ & $0.5625(2)$ & $0.6343(2)$ & $-0.0496(3)$ & $0.070(2)$ & $0.064(2)$ & $0.069(2)$ & $0.007(1)$ & $0.025(1)$ & $0.009(1)$ \\
\hline C(4) & $4 e$ & $0.4312(3)$ & $0.7084(3)$ & $-0.2415(4)$ & $0.058(2)$ & $0.076(3)$ & $0.082(3)$ & $-0.016(2)$ & $-0.005(2)$ & $-0.001(2)$ \\
\hline
\end{tabular}

* Correspondence author (e-mail: karpet@vsibml.mpi-stuttgart.mpg.de) 
Table 3. Continued.

\begin{tabular}{|c|c|c|c|c|c|c|c|c|c|c|}
\hline Atom & Site & $x$ & $y$ & $z$ & $U_{11}$ & $U_{22}$ & $U_{33}$ & $U_{12}$ & $U_{13}$ & $U_{23}$ \\
\hline$C(5)$ & $4 e$ & $0.5078(3)$ & $0.7718(3)$ & $-0.3171(3)$ & $0.051(2)$ & $0.065(2)$ & $0.054(2)$ & $-0.003(2)$ & $0.002(2)$ & $0.000(2)$ \\
\hline$C(6)$ & $4 e$ & $0.5803(2)$ & $0.8438(2)$ & $-0.2485(3)$ & $0.041(2)$ & $0.050(2)$ & $0.047(2)$ & $0.004(1)$ & $0.010(1)$ & $0.000(1)$ \\
\hline$C(7)$ & $4 e$ & $0.6582(2)$ & $0.9145(3)$ & $-0.3160(3)$ & $0.045(2)$ & $0.054(2)$ & $0.042(2)$ & $0.005(1)$ & $0.013(1)$ & $-0.001(1)$ \\
\hline C(8) & $4 e$ & $0.6161(3)$ & $1.0306(3)$ & $-0.3260(3)$ & $0.051(2)$ & $0.056(2)$ & $0.044(2)$ & $0.005(2)$ & $0.006(1)$ & $0.003(1)$ \\
\hline$C(9)$ & $4 e$ & $0.7020(3)$ & $1.1170(3)$ & $-0.3227(3)$ & $0.069(2)$ & $0.054(2)$ & $0.061(2)$ & $0.003(2)$ & $0.019(2)$ & $0.015(2)$ \\
\hline$C(10)$ & $4 e$ & $0.6636(3)$ & $1.0849(3)$ & $-0.1866(3)$ & $0.060(2)$ & $0.048(2)$ & $0.050(2)$ & $0.001(2)$ & $0.013(2)$ & $0.000(1)$ \\
\hline$C(11)$ & $4 e$ & $0.7352(2)$ & $0.9997(3)$ & $-0.0923(3)$ & $0.040(2)$ & $0.054(2)$ & $0.048(2)$ & $-0.004(1)$ & $0.006(1)$ & $0.002(1)$ \\
\hline$C(12)$ & $4 e$ & $0.6593(2)$ & $0.9324(3)$ & $-0.0168(3)$ & $0.045(2)$ & $0.055(2)$ & $0.041(2)$ & $0.001(1)$ & $0.013(1)$ & $0.003(1)$ \\
\hline$C(13)$ & $4 e$ & $0.7701(3)$ & $0.9247(2)$ & $-0.2051(3)$ & $0.045(2)$ & $0.048(2)$ & $0.053(2)$ & $-0.001(1)$ & $0.015(1)$ & $0.008(1)$ \\
\hline$O(13)$ & $4 e$ & $0.8604(2)$ & $0.8831(2)$ & $-0.2064(3)$ & $0.044(1)$ & $0.075(2)$ & $0.081(2)$ & $0.010(1)$ & $0.016(1)$ & $0.004(1)$ \\
\hline$C(30)$ & $4 e$ & $0.5986(3)$ & $0.6151(3)$ & $0.1005(4)$ & $0.106(3)$ & $0.086(3)$ & $0.080(3)$ & $0.005(3)$ & $0.020(3)$ & $0.027(2)$ \\
\hline
\end{tabular}

\section{References}

1. Braun, M.: Photochemische Cycloadditionen von Chloranil und anderen Carbonylverbindungen an Benzvalen, Homobenzvalen, Norbornadien, Norbonen und monocyclische Monoolefine. Dissertation, Universität Würzburg, Germany 1990.

2. Sheldrick, G. M.: Program Package SHELXTL-plus. Release 4.1.

Siemens Analytical X-Ray Instruments Inc., Madison (WI 53719), USA 1990. 